Piccolomini (1405-1463), ou Pie II, évoquent ses « quasi-morts ». Celles-ci relèventelles du miracle ou sont-elles simplement des événements insolites ? Comment le lecteur pourrait-il se prononcer quand le récit évoque l'intervention d'une transcendance dans l'histoire, mais sans pouvoir l'attester autrement que par un acte de foi ? Loin d'offrir des certitudes au lecteur, le récit de cet homme d'Église se trouve pénétré par le suspens (Patricia Eichel-Lojkine). Àl'époque des grandes navigations et des grandes découvertes, et spécialement dans la Venise $\mathrm{du} \mathrm{Xv}^{\mathrm{e}}$ siècle, le voyageur devient parfois narrateur et donne un sens à l'histoire singulière dont il se fait le héros et par laquelle il immortalise l'expérience en récit (Nicole Chareyron). Dans la Vita de Benvenuto Cellini (1558), on remarque une distance ironique entre le narrateur et sa propre narration, même dans les récits de mort : Cellini n'est pas dupe de ses propres hâbleries (Michelle Bianchini). La mort est sans cesse évoquée par les capitaines, auteurs de Mémoires : il en va ainsi dans les Commentaires de Blaise de Monluc (v. 1500-1577) ou dans les Mémoires de Jean d'Antras (1548-1623/27 ?) (Véronique Larcade). À l'inverse du biographe qui connaît la fin de son protagoniste, et qui clôt son récit par la mort de l'individu, l'autobiographe ne peut, quant à lui, raconter sa propre mort. Il lui faut trouver un autre moyen pour terminer son récit. L'étude de quelques Mémoires de la Renaissance met au jour les enjeux et les stratégies de ces clôtures (Nadine Kuperty-Tsur).

Dans un « essai de synthèse » fort utile, Michel Bideaux revient sur les grandes lignes de ce colloque, qui a parfaitement mis en lumière les relations complexes qui existent entre récits de mort et récits de vie.

CHRISTINE PIGNÉ, Université de Caen.

\title{
Bonnes lettres / Belles lettres. Actes des colloques du Centre d'Études et de Recherches Éditer / Interpréter de l'Université de Rouen
} (26 et 27 avril 20oo-6 et 7 février 2003).

Réunis par Claudine Poulouin et Jean-Claude Arnould.

Paris : Honoré Champion, 2006, 486 p.

Sise rue de l'École de Droit, vestige d'un autre âge, l'Académie des Sciences, Arts et Belles-Lettres de Dijon arbore un imposant portail, toujours entr'ouvert. Complaisance envers le touriste moderne, curieux de frôler ce lieu mythique à peu d'égards, n'était le triomphe oratoire du jeune Rousseau contestant la valeur morale des lettres et des arts ? En défendant la thèse d'un effet corrupteur des progrès de l'esprit, Rousseau s'inscrivait en porte-à-faux de l'optimisme des Lumières ; mais 
son célèbre discours (que l'on aurait aimé voir cité dans le volume qui nous intéresse ici) charrie parallèlement deux siècles de débats sur la valeur de ce que l'on peut appeler la civilisation, des Lettres en particulier. Que vaut donc la littérature ? Quelle axiologie sous-tend le qualificatif de « bonnes » ou « belles » attaché aux Lettres ? Le CÉRÉdI de Rouen s'est attaché à poursuivre les tenants et les aboutissants d'une différenciation historique, sans toutefois postuler de réelle solution de continuité ni débattre de l’acte de naissance sans cesse déplacé de la Littérature. Les communications regroupées dans ce volume d'actes (au demeurant doubles : 2000 et 2003) recouvrent la période estampillée « early modern » par les chercheurs anglophones, et le domaine français, de la République des Lettres aux Lettres républicaines. La perspective générale concerne la sociologie de la littérature telle qu'élaborée dans le sillage de La Naissance de l'écrivain : sociologie de la littérature à l'âge classique d'Alain Viala (1985), Des «Belles Lettres » à la « littérature ». Une archéologie des signes du savoir profane en langue française (1680-1760), de Philippe Caron (1992), Littérature et politesse. L'Invention de l'honnête homme 1580-1750 (1996), d'Émmanuel Bury, ou encore Les Pouvoirs de la littérature: histoire d'un paradoxe, de Christian Jouhaud (2000). L'équipe multidisciplinaire rassemblée favorise des approches tantôt littéraires, tantôt historiques, lexicologiques, bibliologiques, socio-politiques, et philosophiques, ce qui résulte en une heureuse complémentarité. Une perspective de surplomb, un corpus colossal et génériquement varié ainsi que des efforts de contextualisation souvent exemplaires devraient attirer un vaste lectorat.

Une histoire de la pensée lettrée à la française induisant maints découpages et recoupements au fil du temps, une évolution en dents de scie se dessine, des bonnes aux belles lettres puis à la littérature, des érudits aux mondains puis aux philosophes et écrivains. Le plan chronologique adopté en témoigne. Les textes et paratextes étudiés au fil des trois grandes sections trahissent des glissements axiologiques et des tensions polymorphes, en résonance avec certaines évolutions institutionnelles et positions polémiques. La première section « Bonnes ou belles lettres » concerne essentiellement le $\mathrm{xVI}^{\mathrm{e}}$ siècle et comprend des essais de Jean-Claude Arnould, Nathalie Dauvois, Hugh Roberts, Tamara Valcic, Witold Konstanty Pietrzak, Yves Delègue, Aline Loicq, et Alain Viala. « Des belles-lettres en question », section centrée autour du Grand Siècle, réunit les communications de Philippe Caron, Déborah Blocker, Jocelyn Royé, Marine Roy-Garibal, Claudine Poulouin, Myriam Maître et Françoise Pélisson-Karo. Enfin, « Des belles-lettres et de la littérature », consacré plus particulièrement au XVIII ${ }^{\mathrm{e}}$ siècle, nous permet de lire Claudine Poulouin (à nouveau), Dinah Ribard, Michèle Rosellini, Carole Dornier, Annie 
Becq, François Bessire, Gérard Gengembre, et Jean-Pierre Cléro. Soit vingt-deux articles au total, dont l'incontournable fil conducteur est l'instabilité sémantique, temporelle, et sociale des notions-sœurs examinées. Un faisceau de dichotomies structure la réflexion : lettres et sciences, raison et goût, plaisir et profit, vertu et beauté ... Quelles disciplines forment les Belles-Lettres ? Histoire, éloquence et poésie s'associent au milieu du $\mathrm{XVII}^{\mathrm{e}}$ siècle en un noyau dur consensuel qui ne doit pas masquer des tensions hiérarchiques sans cesse renouvelées. La place de la fiction narrative, des traductions ou de la philosophie au sein des Belles-Lettres est par exemple discutée.

Quels acteurs et quel public sont concernés ? L'espace social qu'informe ou reflète le débat sur la valeur des lettres évolue, du cabinet aux salons en passant par les écoles ou les académies. La double question de la professionnalisation et du public s'avère récurrente. Noble loisir ou profession ? Patronage ou institutions ? Féminisation symbolique du champ littéraire (Dufour) ? Les acteurs privilégiés de ces changements se distribuent eux-mêmes en factions rivales du métier de penser : public galant contre pédants et doctes (Loicq, Blocker, Dufour), polis et mondains contre froids savants et humanistes attardés (Royé, Roy-Garibal), puis « philosophes » contre les préjugés (Bessire, Gengembre). L'examen de bibliothèques personnelles (Pélisson sur Huet), ou l'activité de grands polygraphes (Blocker sur La Mesnardière, Roy-Garibal sur Furetière, Poulouin), amènent à nuancer ces oppositions fonctionnelles mais réductrices. Le sujet recèle une forte charge socio-politique et partant, polémique. Le rapport au pouvoir de la corporation lettrée (même sous couvert de l'indépendance et de la gratuité aristocratiques) varie suivant les règnes, tout comme l'ascension sociale par le savoir (Loicq, RoyGaribal, Ribard). Une action étatique et des aspirations politiques se font souvent jour (Viala, Rosellini). Le rôle des Académies, dont l'Académie des Inscriptions et Belles-Lettres, fait directement ou indirectement l'objet de plusieurs communications (Loicq, Poulouin, Ribard).

On trouvera en outre des considérations sur l'éducation intellectuelle et morale : pédagogie des lettres, valeur fluctuante de l'exemple (Valcic, Pietrzak, Rosellini), formation par la conversation ou l'érudition, développement de « la critique $\gg$ - que l'on nomme le jugement « goût » ou bien « esprit philosophique » selon l'optique (Caron, Ribard, Rosellini, Bessire). Le problème de la montée en puissance des sciences exactes a également sa place (Caron, Poulouin, Ribard, Cléro sur Pascal, la mathématique et la langue). Une valeur métaphysique, transcendantale du savoir lettré ressort également, les Lettres s'affirmant chez certains auteurs comme un enjeu pour le progrès de l'esprit (individuel et collectif) et la mémoire de l'humanité 
(Arnould sur les préfaces du XVI ${ }^{\mathrm{e}}$ siècle, Poulouin sur l'apologie des Lettres au XVII Gengembre sur Mme de Staël).

Enfin, dernier grand axe de ce copieux recueil, la question de la langue et du style est récurrente, et à bon escient. Il en va d'abord du procès de différenciation entre rhétorique et littérature, puis lettres et sciences - entre (au moins) deux visions concurrentes du bien-dire et du rapport entre beauté d'expression et vertu de l'expression (Gengembre sur Mme de Staël, Cléro pour une philosophie du style). Une pragmatique du discours sous-tend certaines apologies des lettres : si l'on souligne la valeur esthétique de certaines pratiques d'écriture, quel effet, quelle efficacité escompter ou démontrer alors pour gagner voix au chapitre (Dauvois sur la grâce d'Horace, Delègue sur Montaigne, Viala, Rosellini, Becq) ? La vérité historique peut servir de justification (Pietrzak sur historia et fabula). La campagne pour la consécration de la langue vernaculaire passe aussi par le dénigrement du latin des doctes, dans le prolongement de combats humanistes antérieurs (Delègue, Rosellini), et la redéfinition de l'imitation, dans le contexte de la querelle des Anciens et des Modernes notamment (Pélisson, Poulouin). Des approches plus spécifiquement lexicologiques révèlent par ailleurs des aspects polémiques dissimulés ou reflétés par la terminologie.

Parmi les multiples genres et types d'écrits abordés, on rencontrera la poésie, les nouvelles et histoires tragiques (Valcic, Pietrzak), la satire et l'esprit (Roberts sur les sentences cyniques, Royé), la critique, la philosophie (Ribard, Bessire sur Voltaire, Cléro), la didactique (Rosellini sur le manuel de Batteux, Dornier), le roman (Poulouin), et autres traités, registres et dictionnaires. De Ronsard à Voltaire, de Tyard à Madame de Staël, de Montaigne à Pascal, de nombreux auteurs majeurs sont convoqués à côté de fascinants minores. Il manque à cet égard une bibliographie qui rende aisément accessible le large éventail de sources citées, textes rares y compris. Moindre inconvénient étant donné la valeur d'ouvrage de référence de l'ensemble. Au total, de quoi approfondir notre compréhension de cette corporation éclectique et changeante, entre Aristarques et Prométhées — ces « gendelettres » fébrilement honnis par Rimbaud. 